\title{
Characterization of Lipid Nanostructures using DSC coupled to SAXS and WAXS
}

\author{
Daniel J. E. Kalnin \\ YKI - Institute for Surface Chemistry, Box 5607, Stockholm, SE-114 86, Sweden \\ Daniel.Kalnin@Yki.Se
}

\begin{abstract}
Lipids are self-assembling molecules responsible for compartment formation in animal cells. Beside bilayer membranes, they also form all kind of aggregates and mesophases thank to their aptitude to modulate interface curvature. The characterization of the internal structure of such systems is complex and requires the use of combined techniques. Up to recently, droplet size, dilution and complexity prevented any direct identification of the crystalline varieties formed by triacylglycerols inside emulsion globules. Crystallization within this submicron droplets can now be conveniently monitored using Synchrotron X-ray diffraction coupled with DSC. Crystal forms and polymorphism can be influenced by different factors and here we focus on influence factors for crystallization kinetics of emulsified lipids.
\end{abstract}

\section{Keywords:}

Lipid structures, mesophases and biomaterial studies, polymorphism, small angle X-ray scattering, triacylglycerols, multiphase systems,

\section{INTRODUCTION}

Our research focuses mainly on structural properties of lipid self assemblies at a nanometric or even atomic scale. Triacylglycerols (TAGs), the main constituents of fats, exhibit a complex monotropic polymorphism that frequently forecloses the study of thermal and structural properties of the fats.

Polar lipids, such as mono- and diglycerides that are lyotropic and thermotropic show phase transitions of different orders. Naturally lipid structures self-organization into complex structures whose periodicity spans from a few Angstroms up to hundreds of nanometers. Lipids from vegetable or animal origins are widely consumed in food products, for example chocolate, shortenings, margarine and butter but are also used in pharmacy as drug matrices and carrier systems.

The composition of TAGs, which are the main constituents of natural lipids and the physical properties of fat determine the functional properties of fat based matrices.

Since lipids are self-assembling entities they also for lipidbased structures such as Solid Lipid Nanoparticles (SLN), liposomes, cubosomes, isasomes (internal self assembled somes) which are potentially interesting for drug delivery in pharmacy but can also be employed in food science for instance for controlled release or as barrier material.

Studying the properties of lipids is important to better understand and then controlling their physical properties. Increasing the knowledge in both the physical and thermal properties of lipids in situ in food and pharmaceutical products is of tremendous importance with respect to functional, sensorial and textural properties.

Moreover, the increased knowledge of crystallization and polymorphism in lipids has a certain value for technical applications as well as for the development of new processes and food and pharmaceutical products.

The monotropic character of unpolar lipids inducing a polymorphism as well as the lyotropism of polar lipids renders the study of the thermal and structural properties of lipids very complex. Both types of properties are largely depending on sample history, meaning that lipids are thermotropic entities in general.

Their properties are generally determined using thermal methods i.e. differential scanning calorimetry (DSC) and structural methods (e.g. X-ray or neutron diffraction, infrared spectroscopy, etc.). Here we use XRD technique. In this paper we will focus on the physico-chemical properties of emulsified lipids, analyzed by these two techniques coupled and performed on and the same sample, using MICROCALIX calorimeter designed by M. R. Ollivon et al. (e.g. [1-6]). The typical setup of a beam line (Elettra BL 5.2) and sample environment, that allows both small and wide angles X-ray diffraction recordings as well as DSC from one and the same sample is shown below (Figure 1).

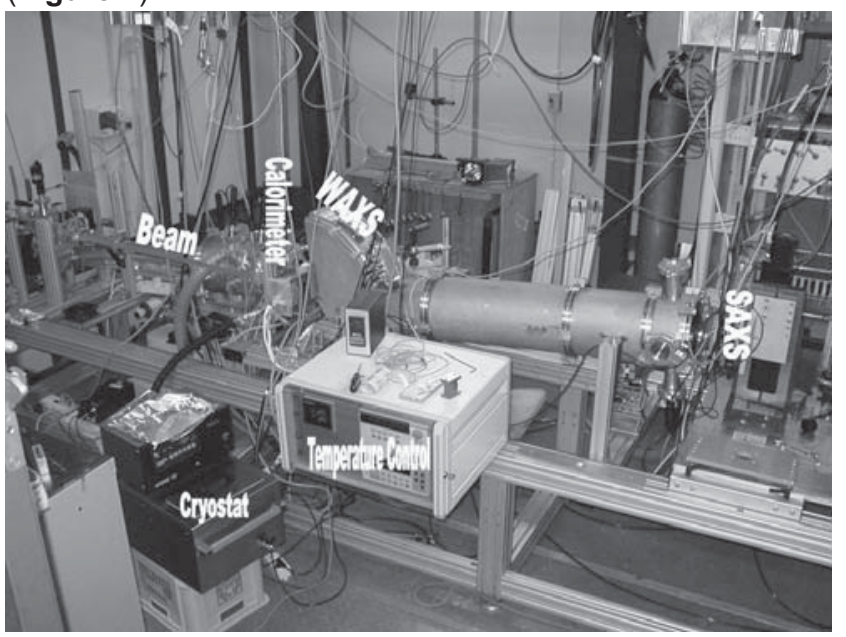

Figure 1: Experimental set-up of MICROCALIX micro calorimeter cell in the time-resolved X-ray diffraction environment used at ELETTRA synchrotron on BL 5.2: (WAXS: linear detector for WAXS-detection, SAXS: linear detector for SAXS-detection, Cryostat: thermostated water bath)

This is an Open Access article distributed under the terms of the Creative Commons Attribution-Noncommercial License (http://creativecommons.org/licenses/by-nc/3.0/), which permits unrestricted use, distribution, and reproduction in any noncommercial medium, provided the original work is properly cited. 


\section{MATERIAL AND METHODS}

\subsection{Material}

Palm oil, based on non hydrogenated lauric oils and free of trans fatty acids, was obtained from Aarhus Utd. (Denmark) and contained $32 \%$ C $12,12 \%$ C14, 24\% C16, 21\% C18:1. The emulsifier, sodium caseinate was industrial food grade (Armor Protéines, Saint Brice en Cogles, France). Sodium azide $\left(\mathrm{NaN}_{3}\right)$ was purchased from Sigma Aldrich.

\section{- Preparation of Model Food Emulsions}

Model food emulsions are prepared one week prior to DSC/XRDT analysis using an APV Lab 2000 homogenizer (Invensys, Evreux, France). Five hundred grams emulsion is prepared, and 500 ppm $\mathrm{NaN}_{3}$ is added to the emulsion. Emulsion is prepared using a two-stage homogenization following a prehomogenization step. First, deionized water (pH 5.5) is heated to $65^{\circ} \mathrm{C}$ and stirred by Polytron PT 3000 (Kinematica, Switzerland) at $5000 \mathrm{rpm}$. During stirring, the temperature is controlled with a thermocouple thermometer and the preparation is covered with Plexiglas to avoid evaporation. Sodium caseinate is added into the aqueous compartment and stirred with the Polytron at $7500 \mathrm{rpm}$ and $\mathrm{T}=60 \pm 5{ }^{\circ} \mathrm{C}$ for $20 \mathrm{~min}$. This step leads to protein hydration and a homogeneous dispersion of the ingredients in the aqueous phase with a $\mathrm{pH}$ of 5.5 in the measurement conditions (Spezialindikator $\mathrm{pH}$ 4.0-7.0 Merck). Then, 20\% heated cocoa butter is added at $65{ }^{\circ} \mathrm{C}$ under stirring at 10 $000 \mathrm{rpm}$ for $5 \mathrm{~min}$ (percentages are expressed as weight percents of the total mass of emulsion). The second step is accomplished in the APV 2000 homogenizer, the two homogenization stages of which are set to 300 ( 10 bar and 50 ( 10 bar, respectively. The homogenizer is insulated with aluminum foil and temperature controlled with water circulation at $65 \pm 5{ }^{\circ} \mathrm{C}$ through a surrounding braided hose. The emulsion is recycled in the homogenizer for $15 \mathrm{~min}$, which is equivalent to five passages of $500 \mathrm{~mL}$ through the homogenizer. The globule size distribution was monitored using laser light scattering until an optimum homogenization time was found. Emulsion is examined by laser light scattering after preparation.

\section{- Laser Light Scattering and Microscopic} Examination

Size distribution of emulsion droplets is determined by laser light scattering using a Malvern Mastersizer (Malvern Instruments Ltd., UK) and is complemented by microscopic examination. Size distribution analysis showed emulsion droplet mean sizes D[4,3] of $0.5 \pm 0.02 \mu \mathrm{m}$.

\section{- Sample Preparations}

The emulsion is aliquoted in $100 \mathrm{~g}$ aliquots and stored at $\mathrm{T}=277 \mathrm{~K}$ in a household refrigerator. Note, prior to analysis, emulsions are stored at $4{ }^{\circ} \mathrm{C}$ for few days $(<7)$. Prior to analysis, as the structure largely depends on sample's time/temperature history, emulsions are heated to $\mathrm{T}=340 \mathrm{~K}$ to equalize thermal history and eliminate all potential nuclei within emulsions' fat droplets which could otherwise influence the emulsions' crystallization behavior.

\subsection{XRD as a function of temperature combined with DSC (DSC/XRDT)}

XRD patterns were recorded with synchrotron radiation on the high-resolution beam line (D22) of L.U.R.E. The X-ray wavelength used was $1.549 \AA$ ( $8 \mathrm{keV})$. Two gas-filled linear detectors (1024 channels, filled with Ar-ethane mixture) were used for data collection using sample-to-detector distances of about $35 \mathrm{~cm}$ and $100 \mathrm{~cm}$ respectively. Both XRD and DSC data were collected and synchronized due to a $\mathrm{C}++$ supported data acquisition system ( $P$. Lesieur). Calibration for XRD was carried out using the form of pure tristearin (StStSt) [7] characterized by a long spacing of $44.98 \AA$ and its higher orders and short spacings of 3.70 , 3.85 and $4.59 \AA$ at room temperature, silver behenate with d-spacings of $58.380 \AA$ [8] and 4-Bromo Benzoic Acid [9] They do perfectly agree but do not cover the same qranges. DSC signal was calibrated for enthalpies and temperatures on melting using lauric acid $(99.5 \%$, $\mathrm{T}_{\mathrm{m}}=316.85 \mathrm{~K} ; \mathrm{H}_{\mathrm{m}}=35.713 \mathrm{~kJ} / \mathrm{mol}$ ) [10]. X-ray diffraction patterns were recorded by transmission using glass capillaries $(0.01 \mathrm{~mm}$ wall thickness $\varnothing=1.5 \mathrm{~mm}$, GLAS W. Müller, Berlin, Germany). Samples were prepared by filling these glass capillaries, with about $20 \mu \mathrm{l}$ of sample, more detailed procedures are given in [1] Emulsion was introduced into capillary at $\mathrm{T}<290 \mathrm{~K}$ (they were stored and sampled at $\mathrm{T}=277 \mathrm{~K}$ using a special syringe) To note, the emulsions analyzed, were carried at $\mathrm{T}=277 \mathrm{~K}$ for the few days following the emulsion preparation.

\section{- Data Analysis}

DSC and XRDT data are collected from the same computer and analyzed to quantify the influence of the constituents in terms of diffracted intensity, peak position, and width. This procedure allows characterization of polymorphic forms as well as of the transitions between them and then identification of DSC thermal events. All data processing is done using IGOR Pro 4.07.

\section{GENERAL MOTIVATION FOR COUPLED EXPERIMENTS- LIPID POLYMORPHISM}

Numerous natural fats are mainly composed of triacylglycerols (TG) which determine their physical and thermal properties [11-13]. Milk and vegetal fats contain a large variety of fatty acids with different chain lengths and degrees of saturation. Due to its complex composition, the melting range of anhydrous milk fat is broad and spans from about -40 to $+40^{\circ} \mathrm{C}$. Furthermore, triacylglycerols are characterized by a complex behavior due to the polymorphism of monotropic type of the solid phases.

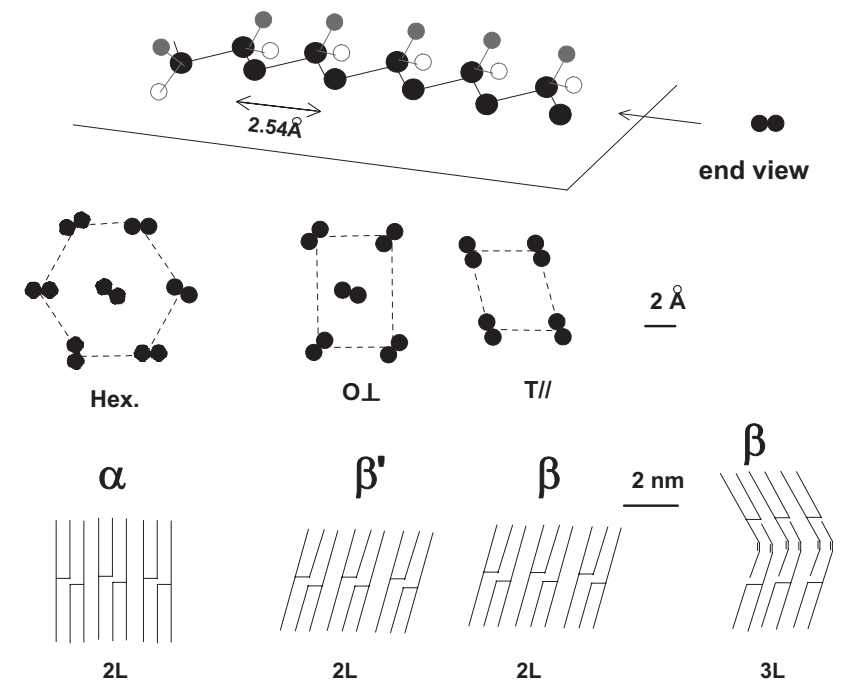

Figure 2: Main types of TG packing. Top, the stable conformation of the hydrocarbon chains of saturated fatty acid is a planar zigzag shown here as a $3 \mathrm{D}$ view along its main axis (note: most hydrogen are not drawn for clarity), right is the end view of the carbon zigzag; Middle, 3 main types of chain lateral packings (hydrogen atoms are not drawn). They allow to define hexagonal (hex.), orthorhombic perpendicular $(\mathrm{O} \perp)$ and triclinic parallel $(\mathrm{T} / /)$ subcells to which $\alpha, \beta$ ', $\beta$ forms correspond. Bottom, 3 main types of TG chain longitudinal packing for trisaturated TG; Bottom right organization of monounsaturated TG of POP type (Fatty acid chains are shown as straight lines) [11-13]. 
Polymorphism of the crystallized phase is a general feature of TG that results from the different possibilities of lateral packing of the hydrocarbon chains and of the longitudinal stacking of molecules in lamellae (Figure 2). These two levels of organization are easily identifiable from the short and long spacings observed by X-ray diffraction at wide and small angles (SAXS/WAXS), respectively [1].

For TG, long spacing's are commonly double or triple chain lengths ( $2 \mathrm{~L}$ or $3 \mathrm{~L}$ ). The short-spacings are characteristic for the type of polymorph, and this has led to the nomenclature given by Larsson that is now widely accepted. The three main types of polymorphic crystal forms in TG are the alpha $(\alpha)$, beta prime $\left(\beta^{\prime}\right)$ and beta $(\beta)$ forms, in the order of their increasing stability. They have been related to different subcells some of them have been described in detail [1423]

Transitions between these three major polymorphic varieties are irreversible. Monotropism implies that they are only possible via a liquid and when leading to the formation of more stable species. TG crystals are made by the stacking of molecule layers, the thickness of which depends on the length and unsaturation of the fatty acid chains, and their angle of tilt with respect to the basal planes formed by the methyl end groups of the TGs. The thickness which can be measured by XRD at small angles $0<\theta<5^{\circ}$, correspond to TG longitudinal stacking and are called "long spacings" (LS). They are primarily related to the number of chains stacked one on top of the other in the main crystalline cell.

For TGs, this number for the LS frequently takes a value of $2(2 \mathrm{~L})$ or $3(3 \mathrm{~L})$, leading to distances in the 40-50 and 55-70 $\AA$ ranges, respectively. Roughly, trilayered (3L) forms are usually related to low-melting, long chain monounsaturated and/or mixed long and short chain TGs, while $2 \mathrm{~L}$ structures are generated mostly by long-chain, high melting, trisaturated TGs. The cross-sectional packings of the aliphatic chains are characterized by specific "short spacings" (SS), independent of chain length and observable at wide angles in the range $8.5<\theta<13^{\circ}$. Short spacings are widely used for identifying the various crystalline subcells (Figure 2, middle) characterizing the polymorphic species. A single line around $4.15 \AA$ characterizes $\alpha$ form, a strong line at $4.6 \AA$ among other sharp lines identifies $\beta$, while $\beta$ ' shows frequently association of two lines among which one is at about $4.2 \AA$ and the other around $3.8 \AA$.

The crystallization and melting of fat are complicated by the occurrence of transitions between several polymorphic crystal forms which depend strongly on heating or cooling rates and on the entire thermal history of the sample [23].

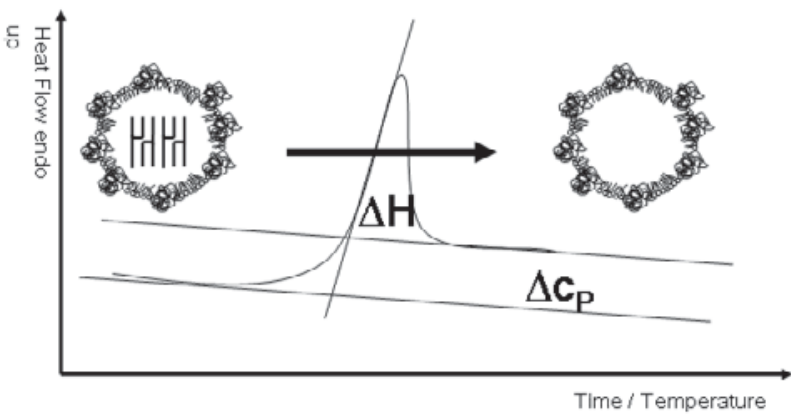

Figure 3: Thermal transitions can be measured yielding information about energy, meaning heat capacity and enthalpy change, however only structural methods can clarify whether the energetic transition is due to the boiling of hot water or just to a fat globule melting in a brick of milk.
For this reason it is important to couple for instance small and wide angle $\mathrm{X}$-ray diffraction with DSC to investigate the thermal properties of emulsified lipids. Until recently, the identification of the crystalline varieties formed by lipids inside the emulsion droplets was not addressed due to the fact that the investigation of emulsions is not only a question of complexity but also a question of concentration of the lipid phase.

\section{RESULTS AND DISCUSSION}

The $20 \%$ palm oil containing emulsion is cooled at $5 \mathrm{~K} / \mathrm{min}$ from $60{ }^{\circ} \mathrm{C}$ to $-10{ }^{\circ} \mathrm{C}$ and successively heated until $40{ }^{\circ} \mathrm{C}$ at $1 \mathrm{~K} / \mathrm{min}$. The cooling at a scanning rate of $5 \mathrm{~K} / \mathrm{min}$ resulting in crystallization of emulsified TAGs monitored by DSC/XRDT is given as an example for coupled experiments (Figure 4). Crystallization as shown by both the exotherm recorded in DSC and the variation of the integrated SAXS diffracted intensity can be unambiguously monitored from $6{ }^{\circ} \mathrm{C}$ on. Most of the TAGs of the emulsified palm oil initially crystallizes in an $2 \mathrm{~L} \alpha$ form characterized by an hexagonal lateral packing of the TAG chains (4.15A, WAXS) and a $2 \mathrm{~L}$ stacking of the whole TAG molecule (41.3Aि, SAXS). It can be deduced that the most of the lipids crystallize while the exothermic event measured by DSC, however it has to be stated that although DSC peak has reached the new baseline (since crystallized emulsion droplets show different $\mathrm{cp}$ ) crystallization is not yet complete and continues until 10 ${ }^{\circ} \mathrm{C}$; limited only by the experimental conditions. It can be concluded that liquid TAG is still co crystallizing inside the confinement of emulsion droplets into $2 \mathrm{~L} \alpha$. No transition has been monitored under cooling conditions as is sometimes the case for emulsified and bulk lipids. At the high scanning rate applied SAXS and WAXS peak appear simultaneously in the same frame together with the DSC signal. The fact that the SAXS peak does merely shift signifies that that co-crystallizing lipids match each other in chain length and inter solubility of the TAG molecules is provided in $2 \mathrm{~L} \alpha$ form.

The beginning of the phase transition from the metastable $2 \mathrm{~L} \alpha$ towards a more stable $\beta^{\prime}$ variety takes place after an induction time at the temperature where the cooling scan is stopped (data not shown). It is up to now not clear whether that would be the case for whatever lowest temperature is used in experimental conditions. It has been shown elsewhere that addition of different emulsifiers can accelerate or retard $2 \mathrm{~L} \alpha \rightarrow \beta^{\prime}$ transitions but no clear rule for the choice of emulsifier has been established. In quiescent conditions no crystallization of the dispersing phase is observed for storage times of up to 45 minutes at $45 \mathrm{~min}$ at $-10^{\circ} \mathrm{C}$

In the shown case, after 10 minutes at $-10^{\circ} \mathrm{C}$ the sample is reheated in order to further induce phase transition. The sample heated at $1 \mathrm{~K} / \mathrm{min}$ shows a progressive $2 \mathrm{~L} \alpha \rightarrow 2 \mathrm{~L} \beta^{\prime}$ phase transition spreading up to $25^{\circ} \mathrm{C}$. The $2 \mathrm{~L} \beta^{\prime}$ is evidenced by diffraction lines at about 4.15 and $3.8 \AA$, with $2 \mathrm{~L}$ stacking of $37.3 \AA$ period (Figure 4 middle). At the molecular level this $2 \mathrm{~L} \alpha \rightarrow 2 \mathrm{~L} \beta^{\prime}$ polymorphic transition is characterized by a change in tilt of the chains perpendicular to the stacking plane. $2 \mathrm{~L} \alpha$ generally present a lower angle of tilt of the TAG molecules than $2 \mathrm{~L} \beta^{\prime}$. This change of tilt is accompanied by a change in lateral chain packing. It is believed that this sort of phase transition is melt mediated; hence nearly no thermal signal is observed. This is due to the fact that even if thermodynamically favored $2 \mathrm{~L} \alpha \rightarrow 2 \mathrm{~L} \beta^{\prime}$ is only driven by the excess enthalpy liberated through the melting of $2 \mathrm{~L} \alpha$ form.

Hence the $2 \mathrm{~L} \alpha \rightarrow 2 \mathrm{~L} \beta^{\prime}$ finishes by the temperature of $25^{\circ} \mathrm{C}$, close to the final melting temperature of $2 \mathrm{~L} \alpha$ form but also the temperature above which no $2 \mathrm{~L} \beta^{\prime}$ crystallize any longer. 


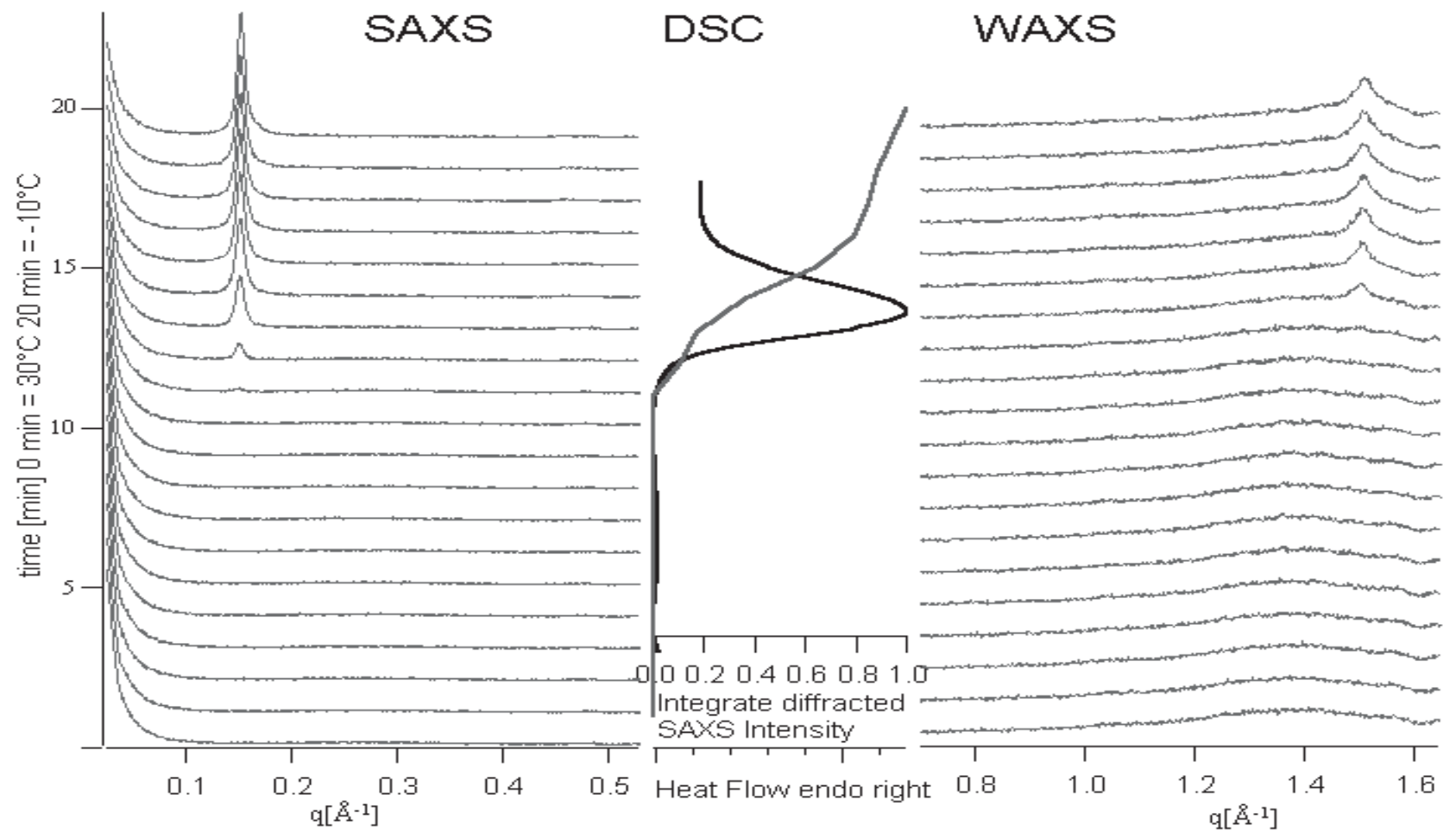

Figure 4 (top): Crystallization of TAGs in an O/W emulsion as followed by simultaneous DSC/XRDT analysis on a cooling scan at $5 \mathrm{~K} / \mathrm{min}$. Left and right: SAXS- WAXS pattern evolutions as a function of time/temperature shows the crystallization of a $2 \mathrm{~L} \alpha$ phase from the melt. Middle: DSC recording is compared to integrated SAXS diffracted intensity of the reflection at $41.3 \AA$.

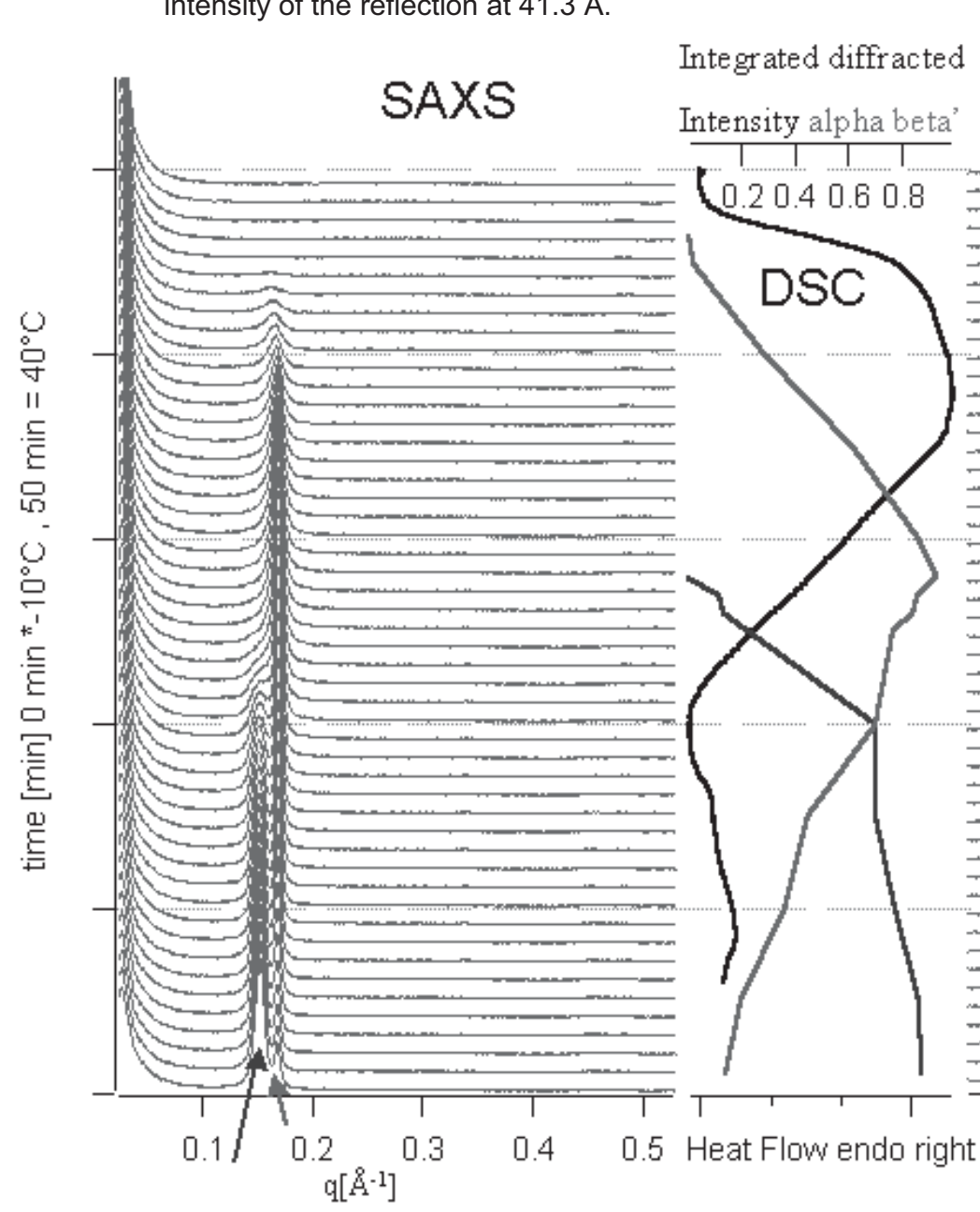

Figure 5 (bottom): Phase transition and final melting of TAGs in an O/W emulsion as followed by simultaneous DSC/XRDT analysis on a heating scan at $1 \mathrm{~K} / \mathrm{min}$. First $2 \mathrm{~L} \alpha \rightarrow 2 \mathrm{~L} \beta^{\prime}$ phase transition takes place upon heating until

$15^{\circ} \mathrm{C}$ where nearly no DSC signal is observed. Final melting of TAGs in an O/W emulsion then follows until $36^{\circ} \mathrm{C}$ by both DSC and XRDT.

\section{WAXS}

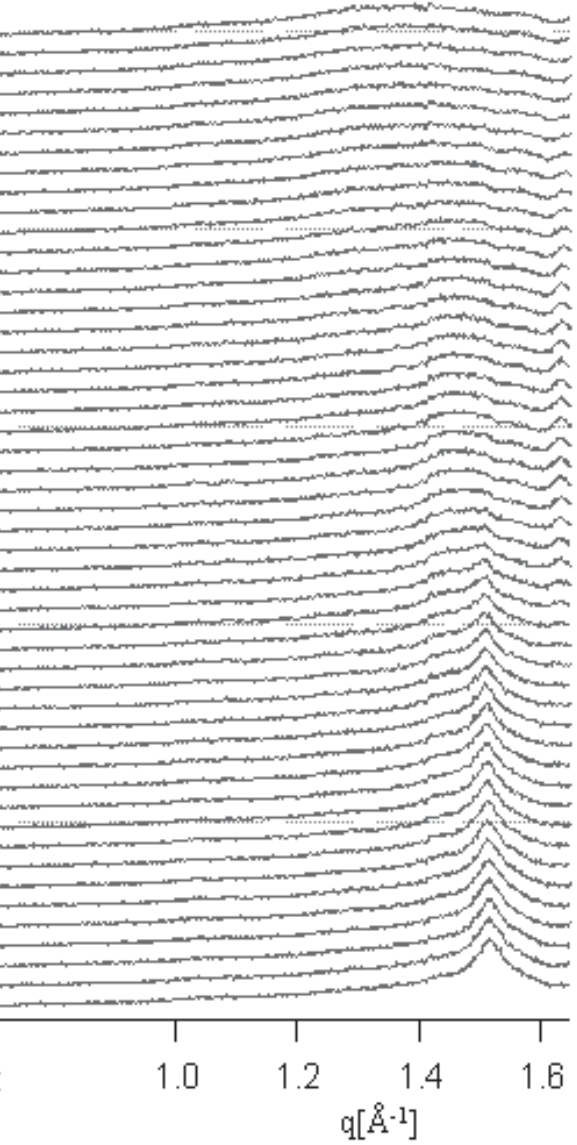


DSC signal upon heating is well correlated with the variations of both integrated SAXS signals. It can be seen that DSC signal upon melting spreads to higher temperatures that both SAXS and WAXS peaks, this fact is either due to the fact that that DSC is more sensitive to small amounts of crystallized matter or due to the fact that XRD peak maximum DSC peak maximum is not correlated and hence shall have different meanings. It is worth noting that the presence of a TAGs liquid phase during the $2 \mathrm{~L} \alpha \rightarrow 2 \mathrm{~L} \beta$ ' phase transition evidenced by a broad bump at $\mathrm{q}$ $=1.35 \AA^{-1}$ never disappears.

Future aims of our work are to determine the respective influences of constituents such as proteins, polar lipids and emulsifiers at the interface upon the TAG crystallization.

\section{SUMMARY}

This paper shows the importance of coupled experiments e.g. in this paper coupling of DSC and XRD. Here it was shown how the use of coupling DSC with XRD enables for evidencing a $2 \mathrm{~L} \alpha \rightarrow 2 \mathrm{~L} \beta^{\prime}$ polymorphic transition which can not be evidenced by thermal techniques alone.

The coupling combines the strong points of both methods and relates hence structural changes to energetically changes. Future method development for coupled techniques will further help to elucidate complex systems especially multi component systems which bear metastable phases.

\section{ACKNOWLEDGMENTS}

D. Kalnin dedicates this work to M. R. Ollivon who unfortunately passed away in 2007 much too soon! I extend my sincere thanks to all who contributed to this work in the group of UMR 8612 especially G. Keller.

\section{REFERENCES}

[1] Keller, G. et al., 1998, DSC and X-ray diffraction coupling. Specifications and applications, J. Thermal Anal. 51, 783-791.

[2] KALNIN D. et al., 2004), Fat Crystallization in Emulsion: Influence of Emulsifier Concentration on Triacylglycerol Crystal Growth and Polymorphism, Crystal Growth \& Design, 4, 6, 1283-1293.

[3] Lopez, C et al., 2001, Thermal and Structural Behavior of Milk Fat : 2. Crystalline Forms Obtained by Slow Cooling of Cream, J. Colloid Interface Sci., 240 150-161.

[4] Kalnin D. et al., 2005, Systematic investigation of lard polymorphism using combined DSC and time resolved synchrotron X-ray diffraction, Eur. J. Lipid Sci. Tech., 107, 9, 594-606.

[5] Kalnin, D. et al., 2002, Monitoring fat crystallization in aerated food emulsions by combined DSC and timeresolved synchrotron X-ray Diffraction, Food Res. Int. $35,927-934$
[6] Kalnin, D. et al., 2009, Structural behaviour of lipid droplets in protein-stabilized nano-emulsions of alpha tocopherol, Food Biophysics, 3, 2 163-168.

[7] Ollivon, M., 1982, Ph.D. thesis, Université Pierre et Marie Curie.

[8] Huang, T. C. et al, 1993, X-ray powder diffraction analysis of silver behenate, a possible low angle diffraction standard, J. Appl. Crystallogr. 26, 180-184.

[9] Ohkura, K. et al., 1972 The Crystal and Molecular structure of p-Bromobenzoic Acid, Bull. Chem. Soc. Jpn. 45, 8, 2651-2652.

[10] Grabielle Madelmont C. et al., 1983, Calorimetric Studies on Phospholipid-Water Systems I. DLDipalmitoylphosphatidylcholine (DPPC) - Water System, Journal of Colloid and Interface Science, 95 No. 2, 471- 482

[11] Larsson, K., 1986, In The Lipid Handbook; Gunstone, D., Harwoood, J. L., Padley, F. B., Eds.; Chapman \& Hall: London, Chapter 8, pp 321-384.

[12] Small, D. M., 1986, In Handbook of Lipid Research. The Physical Chemistry of Lipids. From Alkanes to Phospholipids; Plenum Press: New York.

[13] Ollivon, M. et al., 1996; In Oils and Fats Manual; Karleskind, A., Wolff, J. P., Eds.; Lavoisier: Paris, Vol 1.

[14] Larsson, K., 1966, Classification of Glyceride Crystal Forms, Acta Chem. Scand., 20, 2255-2260.

[15] Hernquist, L. et al., 1982, On the crystal structure of the $\beta^{\prime}$ form of triglycerides and structural changes at the phase transitions Liq. $->\alpha->\beta^{\prime}->\beta$, Fette Seifen Anstrichm. 84, 349-354.

[16] Dohi, K. et al., 2002, X-ray and vibrational spectroscopy studies on polymorphism of trielaidin, J. Cryst. Growth, 2227-2232.,

[17] Larsson, K., 1973, Molecular arrangements in Glycerols, Fette Seifen Anstrichm. 74, 136 - 142.

[18] Sato, K. 2001, Crystallization behaviour of fats and lipids a review, Chem. Eng. Sci. 56, 2255-2265.

[19] Gibon, V. et al., 1984, New data about Molecularstructure of beta Trilaurin, Bull. Soc. Chim. Belg. 93, 27-34

[20] Sato, K. et al., 1989, Polymorphism of POP and SOS III. Solvent crystallization of $\beta_{2}$ and $\beta_{1}$ polymorphs, J. Am. Oil Chem. Soc. 66, 664-674.

[21] van Langevelde, A. et al., 2000, Structure of $\mathrm{CnCn}+2 \mathrm{Cn}$ type ( $\mathrm{n}=\mathrm{e}$ even) beta' triacylglycerols, Acta Crystallogr., Sect. B: Struct. Sci. 56, 1103-1111.

[22] Larsson, K., Ed. Lipids: Molecular Organization, Physical Functions and Technical Applications; The Oily Press: Dundee, Scotland, 1994; Vol. 5.

[23] Ollivon, M. et al., 1982, Measurement of enthalpies and entropies of unstable crystalline forms of saturated even monoacid triglycerides, Thermochim. Acta, 53, 183-194. 